\title{
PRODUÇÃO DE BIOSSURFACTANTES E DETERMINAÇÃO DE SUAS ATIVIDADES DE DESEMULSIFICAÇÃO
}

\author{
B.A.C. ROQUE ${ }^{1}$, P.P.F. BRASILEIRO ${ }^{1}$, AA.L. PINTO ${ }^{1}$, J.M. LUNA ${ }^{1,2}$, L.A. SARUBBO ${ }^{1,2}$, R.D. \\ RUFINO $^{1,2}$ \\ ${ }^{1}$ Universidade Católica de Pernambuco, Centro de Ciências e Tecnologia; \\ ${ }^{2}$ Centro de Gestão de Tecnologia e Inovação (CGTI) \\ E-mail para contato: raqueldrufino@yahoo.com.br
}

\begin{abstract}
RESUMO - Emulsões estáveis são geradas em diversos ramos industriais. Os desemulsificantes utilizados pela indústria petrolífera são compostos tóxicos. Dessa forma, os biossurfactantes surgem como agentes promissores por serem biodegradáveis. No presente estudo, foram produzidos biossurfactantes por diferentes espécies de microorganismos, e determinadas suas características básicas. Após essa etapa, os biopolímeros foram selecionados para determinação de suas atividades de desemulsificação. Os resultados mostraram que os biossurfactantes produzidos pela Pseudomonas aeruginosa e Candida lipolytica apresentaram valores de tensão superficial de $26 \mathrm{mN} / \mathrm{m}$ e de $4,2 \mathrm{~g} / \mathrm{L}$ e 0,3 g/L para suas Concentrações Micelares Críticas, respectivamente. Nos demais testes o biopolímero produzido pela levedura apresentou taxas de desemulsificação de $60 \%$ para a desestabilização de emulsões modelo água em óleo. Dessa forma, os biossurfactantes selecionados apresentaram potencial de utilização como desemulsificantes.
\end{abstract}

\section{INTRODUÇÃO}

Uma emulsão é um sistema termodinamicamente instável entre dois ou mais líquidos imiscíveis, onde uma fase engloba outra pela diferença entre as forças repulsivas de cada gotícula. Dependendo do disposição do líquido na fase contínua, as emulsões são classificadas como água-emóleo (A/O) ou óleo-em-água (O/A). E podem ser geradas em diversas empresas como as de aço, alumínio, alimentos, têxteis, couro, produtos petroquímicos, indústrias de acabamento de metais entre outras (Larson et al., 1994).

Os desemulsificantes utilizados pela indústria petrolífera são compostos de um ou mais agentes tensoativos derivados de ácidos graxos, glicóis e alquilfenóis dispersos em um sistema de solvente. Além de conterem principalmente esses compostos, têm efeitos contaminantes em organismos marinhos e não atendem aos níveis de biodegradação desejáveis. Posteriormente, é provável que a maioria dos desemulsificantes convencionais terão uso proibido, utilizando como alternativa a estabilização de óleo-em-água por polímeros solúveis, a qual tem sido amplamente investigada tanto do ponto de vista teórico como experimental (Lee; Lee 2000). 
Os surfactantes são agentes anfipáticos de grandes aplicações industriais. Muitos tipos de surfactantes quimicamente sintetizados são utilizados, embora o desenvolvimento de produtos alternativos, biodegradáveis e menos tóxicos, como os biossurfactantes, agentes produzidos por vias microbiológicas, torna-se uma estratégia importante na obtenção de componentes mais compatíveis com o meio ambiente e na ampliação das propriedades específicas e aplicações desses compostos (Souza Sobrinho et al., 2013).

Dessa forma, os biossurfactantes surgem como agentes promissores devido a sua origem microbiológica. Essas biomoléculas têm se destacado como tecnologia alternativa no controle da poluição provocada por hidrocarbonetos do petróleo (Rufino et al., 2014). Comparados com os surfactantes sintéticos, os derivados do petróleo, os biossurfactantes, em geral, exibem forte compatibilidade ambiental, menor tensão superficial, baixa toxicidade e alta biodegradabilidade, além de serem produzidos por fontes renováveis (Luna et al., 2013).

A tensão superficial é a força de atração, existente entre as moléculas dos líquidos com o ar. Essa força repulsiva diminui quando a concentração do surfactante no meio aquoso aumenta, ocorrendo a formação de micelas, que são agregados moleculares, possuindo ambas as regiões estruturais hidrofílica e hidrofóbica. Esse caráter anfipático permite a associação dinâmica espontaneamente em solução aquosa a partir de certa concentração micelar crítica (CMC), formando grandes agregados moleculares de dimensões coloidais. Esta concentração corresponde à mínima concentração de surfactante necessária para que a tensão seja reduzida ao máximo (Rufino et al., 2014).

Nesse trabalho, os agentes surfactantes produzidos por diferentes espécies de leveduras e bactérias cultivadas em resíduos industriais foram produzidos e suas propriedades básicas determinadas para utilização em experimentos futuros de determinação de suas atividades de desemulsificação, tendo em vista aplicações futuras na dispersão de emulsões produzidas por diversos setores industriais.

\section{METODOLOGIA}

\subsection{Micro-organismos Produtores}

Os micro-organismos utilizados para a produção dos biossurfactantes foram: Candida guilliermondii, Candida lipolytica, Pseudomonas cepacia, Pseudomonas sp. e Pseudomonas aeruginosa, depositadas no Banco de Culturas do Núcleo de Pesquisa em Ciências Ambientais (NPCIAMB) da Universidade Católica de Pernambuco. 


\subsection{Meios de Produção}

Biossurfactante A: água destilada, contendo as fontes de carbono (óleo de fritura residual $2,5 \%$; melaço - 2,5\%) e nitrogênio (milhocina - 4,0\%).

Biossurfactante B: meio mineral descrito por Rufino et al., (2014), acrescido de 6\% de resíduo da refinaria de óleo de soja e $1 \%$ de ácido glutâmico.

Biossurfactante C: meio mineral descrito por Dubey e Juwarkar (2004), acrescido das fontes de carbono (óleo de canola residual de fritura -2,0\%) e nitrogênio (milhocina -3,0\%).

Biossurfactante D: meio mineral descrito por Bushnell e Hass (1941) acrescido das fontes de carbono (melaço de cana) e nitrogênio (milhocina) nas concentrações de 3,0\% cada.

Biossurfactante E: água destilada, contendo as fontes de carbono (resíduo da refinaria de óleo de soja $-4,0 \%$ ) e nitrogênio (milhocina - $0,5 \%$ ).

\subsection{Isolamento dos Biossurfactantes}

Biossurfactante A: o isolamento do biossurfactante produzido por $C$. guilliermondii ocorreu pela centrifugação a $3500 \mathrm{rpm}$ durante 20 minutos para retirada das células do líquido metabólico, sendo submetido ao processo de extração. $\mathrm{O} \mathrm{pH}$ foi ajustado para $2 \mathrm{com}$ solução de $\mathrm{HCl} 6,0 \mathrm{M}$ e precipitado com 2 volumes de metanol (Souza Sobrinho, 2007).

Biossurfactante B: o biossurfactante produzido por $C$. lipolytica foi isolado de acordo com Rufino et al., (2013).

Biossurfactante C, D e E: os isolamentos dos biossurfactantes produzidos por diferentes espécies de Pseudomonas foram realizados de acordo com Costa et al. (2006).

\subsection{Determinação das Propriedades Tensoativas dos Biossurfactantes}

A tensão superficial e a Concentração Micelar Crítica (CMC) foram determinadas em tensiômetro automático KSV Sigma 700 (Finland) utilizando-se o anel de NUOY.

\subsection{Preparação das Emulsões Modelo Água/Óleo (A/O) e Óleo/Água (O/A)}

Emulsões preparadas pela adição das fases oleosas (querosene) e aquosa, contendo 0,01\% $(\mathrm{v} / \mathrm{v})$ de emulsificantes e agitadas em homogeneizador a $15000 \mathrm{rpm}$ por 5 minutos. A razão 
volumétrica de fase oleosa para fase aquosa foi de 6:4 (Cairns et al., 1982). Para preparar a emulsão modelo O/A, $200 \mathrm{~mL}$ de querosene contendo 0,8gL de Span 80 e $300 \mathrm{~mL}$ de água deionizada contendo $1 \mathrm{gL}$ de Tween 80 foram misturados a $15000 \mathrm{rpm}$ por 5 minutos (Nadarajah et al., 2002).

A emulsão modelo A/O foi obtida pela mistura de $300 \mathrm{~mL}$ de querosene contendo $16,7 \mathrm{gL}$ de Span 80 com 200mL de água deionizada 15000 rpm por 5 minutos (Huang et al., 2009).

O desempenho da desemulsificação foi avaliado calculando-se a taxa de desemulsificação como mostrado abaixo:

$$
\text { Demulsificação }(\%)=\left[1-\frac{\text { volume da emuls ão remanescente }}{\text { volume total da emuls ão }+ \text { volume de amostra adicionada }}\right] \times 100
$$

\subsection{Teste de Desemulsificação para Emulsões com Óleo Motor}

Para o teste de desemulsificação, $2 \mathrm{~mL}$ do líquido metabólico livre de células contendo cada biossurfactante foi adicionado a tubo graduado contendo $18 \mathrm{~mL}$ da emulsão de óleo de motor e água na proporção de 1:1 (v/v). Um branco contendo $2 \mathrm{~mL}$ do meio de produção estéril foi usado, bem como um tubo contendo apenas a emulsão óleo de motor/água. Os tubos foram, então, deixados em repouso à temperatura ambiente. A alteração do volume da fase aquosa foi registrada após 24 horas. O desempenho da desemulsificação também foi avaliado, calculando-se a taxa de desemulsificação como mostrado anteriormente.

\section{RESULTADOS E DISCUSSÃO}

\subsection{Determinação das Propriedades Tensoativas dos Biossurfactantes}

As propriedades tensoativas de um surfactante dependem principalmente de sua habilidade em reduzir as tensões superficial e interfacial, dos seus valores de CMC e da formação de emulsões compactas e estáveis (Santos et al., 2013).

A capacidade de reduzir a tensão superficial depende da concentração do composto tensoativo, isto é, da CMC, que é definida como a concentração mínima do surfactante requerida para reduzir ao máximo a tensão superficial da água, dando início à formação de micelas. Surfactantes eficientes possuem valores de CMC muito reduzidos, o que significa que pouco surfactante é requerido para reduzir a tensão superficial. Para fins práticos, é importante fazer a distinção entre um surfactante eficiente e um surfactante eficaz. A eficiência é medida pela concentração de surfactante requerida para produzir uma redução significativa da tensão superficial da água, enquanto que a eficácia é medida pelo valor mínimo que a tensão superficial pode atingir (Silva et al, 2010). A Tabela 1 apresenta os resultados das principais propriedades dos biossurfactantes produzidos por diferentes micro-organismos em meios de produção de baixo custo. 
Tabela 1 - Determinação das propriedades dos biossurfactantes produzidos por Candida guilliermondii (A), Candida lipolytica (B), Pseudomonas cepacia (C), Pseudomonas sp. (D) e Pseudomonas aeruginosa (E)

\begin{tabular}{ccc}
\hline BIOSSURFACTANTE & $\begin{array}{c}\text { TENSÃO SUPERFICIAL } \\
(\mathbf{m N} / \mathbf{m})\end{array}$ & $\begin{array}{c}\text { CONCENTRAÇÃO } \\
\text { MICELAR CRÍTICA (CMC) } \\
(\mathbf{g} / \mathbf{L})\end{array}$ \\
\hline A & 31 & 4,2 \\
B & 25 & 0,3 \\
C & 28 & 16 \\
D & 29 & 5 \\
E & 26 & 6 \\
\hline
\end{tabular}

Os biossurfactantes produzidos por diferentes micro-organismos apresentaram resultados eficientes na redução da tensão superficial do meio de cultivo, sendo os biopolímeros produzidos por Candida lipolytica e Pseudomonas aeruginosa os que demonstraram as maiores ações na redução da tensão superficial do meio de produção, com valores de 25 e 26 mN/m, respectivamente. Em relação aos resultados observados para a Concentração Micelar Crítica (CMC) os biossurfactantes produzidos pelas leveduras C. guilliermondii e C. lipolytica apresentaram valores de 4,2 e 0,3 g/L, respectivamente.

Santa Anna et al. (2002), estudando a produção de biossurfactante por $P$. aeruginosa PA1 cultivada em meio mineral, contendo 3\% de glicerol observaram uma CMC de $190 \mathrm{mg} / \mathrm{L}$ para uma tensão superficial de $27 \mathrm{mN} / \mathrm{m}$. Os valores de CMC de misturas de surfactantes obtidos do cultivo de $P$. aeruginosa LB1 em óleos nativos brasileiros variaram de 29,8 a 31,5 mN/m, com CMC entre 55 e $163 \mathrm{mg} / \mathrm{L}$ (Costa et al., 2006). Já o biossurfactante de $P$. aeruginosa Bs20 cultivada em óleo de soja reduziu a tensão superficial da água para $30 \mathrm{mN} / \mathrm{m}$ a uma concentração de 13,4 mg/L (AbdelMawgoud et al., 2009).

O biossurfactante produzido por $P$. putida 300-B cultivada em óleos de fritura e glicose,, apresentou uma CMC de $91 \mathrm{mg} / \mathrm{L}$ e tensão superficial de 31,2 m/Nm (Raza et al., 2007). Valores de CMC em torno de $230 \mathrm{mg} / \mathrm{L}$ foram encontrados para o biossurfactante de $P$. aeruginosa AT10 cultivada em óleo de soja (Haba et al., 2003).

Vários micro-organismos podem ser utilizados para modificar as propriedades de uma emulsão, usando superfícies celulares hidrofóbicas ou a natureza anfipática dos biossurfactantes, para deslocar ou modificar emulsionantes presentes na interface óleo-água (Das, 2001). As bactérias pertencentes ao gênero Nocardia, Corynebacterium, Rhodococcus, Mycobacterium, Bacillus, são exemplos de micro-organismos produtores de biossurfactantes, que apresentem atividade de desemulsificação (Liu et al., 2011). Os desemulsificantes microbianos apresentam baixa toxicidade, biodegradabilidade e alta eficiência em condições ambientais extremas. Além disso, a emulsão de hidrocarbonetos pode 
garantir o crescimento microbiano, e o crescimento pode ocorrer também simultaneamente com a desemulsificação (Kosaric et al., 1987).

Com base nos resultados observados, os biossurfactantes produzidos por Candida lipolytica, C. guilliermondii e Pseudomonas aeruginosa foram selecionados para estudos posteriores de determinação das suas atividades de desemulsificação.

\subsection{Determinação das Atividades de Desemulsificação}

Os desemulsificantes microbianos possuem baixa toxicidade, biodegradabilidade e alta eficiência sob condições estremas (Rocha e Silva et al., 2013). Testes para determinação das atividades de desemulsificação foram realizados para os biossurfactantes selecionados na etapa anterior.

Os experimentos de desemulsificação foram conduzidos de acordo com Liu et al. (2009) em valores de $\mathrm{pH}$ que variaram de 6,0 a 6,8. Os resultados obtidos demonstraram que taxas de desemulsificação de 60,15\% para emulsões modelo do tipo A/O (Água em Óleo) e 37\% de taxa de desemulsificação para emulsões contendo óleo de motor e água e $21,9 \%$ de taxa de desemulsificação para emulsões contendo óleo de motor e água do mar.

Shin et al. (2008) enfatiza que o aumento de pH é essencial para a potencialização da atividade desemulsificante, pois o efeito da alteração dessa propriedade pode ser associado à manipulação fisico-química da performance do biossurfactante. A exposição a ambientes alcalinos torna os biossurfactantes mais hidrofílicos, facilitando o processo de desemulsificação da emulsão.

\section{Conclusões}

Dessa forma os biossurfactantes testados apresentaram resultados satisfatórios em relação as suas propriedades tensoativas, e os demais testes demonstraram percentuais elevados para as taxas de desemulsificação das emulsões contendo óleo motor. Sendo assim, pode-se sugerir o potencial futuro de aplicação dessas biomoléculas no controle da poluição ambiental causada por emulsões vindas de diversos ramos industriais.

\section{REFERÊNCIAS}

ABDEL-MAWGOUD, A.M.; ABOULWAFA, M.M.; HASSOUNA, N. A-H. Characterization of rhamnolipid produced by Pseudomonas aeruginosa isolate Bs20. Applied Biochemistry and Biotechnology, DOI 10.1007/s12010-008-8285-1, 2008.

BUSHNELL, L.D.; HASS, H.E. The utilization of certain hydrocarbons by microorganisms. Journal of Bacteriology, v. 41, p. 653-658, 1941. 


\section{9 a 22 de outubro de 2014 \\ Florianópolis/SC}

CAIRNS, W.L.; COOPER, D.G.; ZAJIC, J.E.; WOOD, J.M.; KOSARIC, N. Characterization of Nocardia amarae as potent biological coalescing agent of water-in-oil emulsions. Appl. Environ. Microbiol., v. 43, p. 362-366, 1982.

COSTA, S.G.V.A.O.; NITSCHKE, M.; HAADDAD, R.; ERBELIN, M.N.; CONTIERO, J. Production of Pseudomonas aeruginosa LBI rhamnolipids following growth on Brazilian native oils. Process Biochemistry, v. 21, p. 1593-1600, 2006.

DAS, M. Characterization of de-emulsification capabilities os a Micrococus sp. Bioresource Technology, v. 79, p. 15-22. 2001.

DUBEY, K., JUWARKAR, A. Distillery and curd whey wastes as viable alternative sources for biosurfactant production. World Journal of Microbiology and Biotechnology, v. 17, p. 61-69, 2001.

HABA, E.; ABALOS, A.; JÁUREGUI, O.; ESPUNY, M.J.; MANRESA, A. Use of Liquid Chromatography-Mass Spectroscopy for Studying the Composition and Properties of Rhamnolipids Produced by Different Strains of Pseudomonas aeruginosa. Journal of Surfactants and Detergents, v. 6, p. 155-161, 2003.

HUANG, X.F.; LIU, J.; LU, L.J.; WEN, Y.; XU, J.C.; YANG, D.H.; ZHOU, Q. Evaluation of screening methods for demulsify bactéria and characterization of lipopeptide bio-emulsifer produced by Alcaligenes sp. Bioresour. Technol. v.100, p. 1358-1365. 2009.

KOSARIC, N.; CAIRNS, W.L.; GRAY, N.C.C. Microbial emulsifiers and de-emulsifiers. In: KOSARIC, N.; CAIRNS, W.L.; GRAY, N.C.C. eds. Biosurfactants and biotechnology. Surfactant Science Series, v. 25, Dekker, New York, p.247-331, 1987.

LARSON, K.; RAGHURAMAN, B.; WIENCEK, J. Electrical and chemical deemulsification techniques for microemulsion liquid membranes. Journal of Membrane Science, v. 91, p. 231248, 1994.

LEE, J.C.; LEE, K.Y. Emulsification using environmental compatible emulsifiers and deemulsification using DC field and immobilized Nocardia amarae. BiotechnologyLetters, v. 22, p. 1157-1163, 2000.

LUNA, J.M.; RUFINO, R.D.; SARUBBO, L.A.; CAMPOS-TAKAKI, G.M. Characterization, surface properties and biological activity of a biosurfactant produced from industrial waste by Candida sphaerica UCP0995 for application in the petroleum industry. Colloids and Surfaces B: Biointerfaces. v. 102, p. 202-209. 2013.

LIU, J.; LU, L. J.; HUANG, X. F.; SHANG, J. J.; LI, M. X.; XU, J. C.; DENG, H. P. Relation ship between surface physico chemical properties and its demulsifying ability of na alkaliphilic strain of alcaligenes sp. S-XJ-1. Process Biochem. v.46, p.1456-1461. 2011 


\section{9 a 22 de outubro de 2014 \\ Florianópolis/SC}

LIU, J.; HUANG, X. F.; LU, L. J.; XU, J. C.; WEN, Y.; YANG, D. H. Comparison between waste frying oils and paraffin as carbono source in the production of biodemulsifier by dietzia sp. SJS-1. Beoresource Technology, v.24, n. 100, p. 6481-9487, 2009.

NADARAJAH, N. Evaluation of a mixed bacterial culture for the de-emulsification of water-in oil petroleum emulsions. Msc. Thesis, University of Waterloo, Canada. 2002.

RAZA, Z.A.; KHALID, Z.M.; KHAN, M.S.; BANAT, I.M.; REHMAN, A.; NAEEM, A.; SADDIQUE, M.T. Surface properties and sub-surface aggregate assimilation of rhamnolipid surfactants in different aqueous systems. Biotechnol. Lett. v. 32, p. 811-816. 2010.

ROCHA E SILVA, F.M.P.; ROCHA E SILVA, N.M.P.; RUFINO, R.D.; LUNA, J.M.; SANTOS, V.A.; SARUBBO, L.A. Avaliação de biossurfactantes como desestabilizantes de emulsões com hidrocarbonetos. VI Simpósio de Ciências Biológicas (SIMCBIO). Universidade Católica de Pernambuco, Recife, PE. 2013.

RUFINO, R.D.; LUNA, J.M.; CAMPOS-TAKAKI, G.M.; SARUBBO, L.A. Characterization and properties of the biosurfactant produced by Candida lipolytica UCP 0988. Electronic Journal of Biotechnology. v. 17, p. 34-38, 2014.

SANTA ANNA, I.M.; SEBASTIAN, G.V.; PEREIRA JR., N.; ALVES, T.L.M.; MENEZES, E.P.; FREIRE, D.M.G. Production of biosurfactant from a new andnpronussing strain of Pseudomonas aeruginosa P.A.1. Appl Biochem Biotechnol, v. 91, p. 459-467, 2007.

SANTOS, D.K.F.; RUFINO, R.D.; LUNA, J.M.; SANTOS, V.A.; SALGUEIRO, A.A.; SARUBBO, L.A. Synthesis and evaluation of biosurfactant produced by Candida lipolytica using animal fat and corn steep liquor. Journal of Petroleum Science and Engineering, v. 105, p. 43-50. 2013.

SHIN, K. H.; KIM, K. W.; KIM, J. Y.; LEE, K. E. HAN, S. S. Rhamnolipid morphology and phenanthrene solubility at different pH values. J. Environ. Qual. v.37, p.509-514, 2008.

SILVA, S. N. R. L.; FARIAS, C. B. B.; RUFINO, R. D.; LUNA, J. M.; SARUBBO, L.A. Glycerol as substrate for the production of biosurfactant by Pseudomonas aeruginosa UCP0992. Colloids and Surfaces B: Biointerfaces, v.79, p.174-183, 2010.

SOUZA SOBRINHO, H.B.S.; RUFINO, R.D.; LUNA, J.M.; SALGUEIRO, A.A.; CAMPOSTAKAKI, G.M.; LEITE, L.F.C.; SARUBBO, L.A. Utilization of two agroindustrial by-products for the production of a surfactant by Candida sphaerica UCP0995. Process Biochemistry, v. 43, p. 912-917. 2008.

SOUZA SOBRINHO, H.B.S.; LUNA, J.M.; RUFINO, R.D.; PORTO, A.L.F.; SARUBBO, L.A. Application of biosurfactant from Candida sphaerica UCP 0995 in removal of petroleum derivate from soil and sea water. Journal of Life Sciences, v. 7, p. 559-569. 2013. 